\title{
TRADUCCIÓN
}

\section{LA ALFABETIZACIÓN INFORMACIONAL COMO DERECHO HUMANO*}

\author{
Paul Sturges
}

\section{Almuth Gastinger ${ }^{* *}$}

Resumen: Se puede argumentar que existe una relación entre el derecho a la libertad intelectual (argumento pasivo) propuesto por el Artículo 19 de la Declaración Universal sobre los Derechos Humanos de las Naciones Unidas y la consecuente responsabilidad por parte de los gobiernos, los profesionales y los defensores de los derechos civiles de crear (argumento activo) las condiciones idóneas para el ejercicio eficaz de la libertad intelectual. Cada vez más los analistas del papel de los medios de comunicación en la sociedad y de la responsabilidad social de la informática también están llegando a conclusiones de este tipo, resaltando la importancia tanto de la alfabetización mediática como de la alfabetización en informática. Obviamente, este argumento nos lleva a analizar el planteamiento que los profesionales de la información se hacen de los cursos sobre alfabetización de información que actualmente imparten. Aunque existen muchos programas de alfabetización informacional que son muy completos, éstos, hasta ahora, se basan, en su mayor parte, en lo que los profesionales piensan que son las necesidades prácticas de los usuarios. Sin embargo, se puede interpretar que las declaraciones de tipo general como, por ejemplo, la Declaración de Praga "Hacia una Sociedad de la Alfabetización Informacional” del 2003 y la Proclamación de Alejandría del 2005 empiezan a apuntar hacia una justificación de las actividades de la alfabetización informacional basadas en los derechos humanos, en concreto en el artículo 19. El argumento es que si se parte de una perspectiva basada en los derechos humanos podemos llegar a una interpretación sólida de la alfabetización informacional que incluya la alfabetización mediática, la alfabetización en informática, la alfabetización en la web y, hasta cierto punto, la alfabetización cívica, desarrollando de este modo un modelo que responde a las necesidades humanas más que a las prioridades establecidas por los profesionales de la información. En este artículo se destaca el valor de este planteamiento tanto en la práctica como para la investigación.

Palabras clave: Alfabetización informacional; derechos humanos; libertad intelectual.

Title: INFORMATION LITERACY AS A HUMAN RIGHT.

\footnotetext{
* Traducción del artículo "Information Literacy as a Human Right”, de Paul Sturges y Almuth Gastinger, publicado en la revista Libri, 2010, vol. 60, n 3, p. 195-202.

** Traducido por Lozano Palacios, A. en colaboración con alumnos de la Facultad de Comunicación y Documentación de la Universidad de Granada.

STURGES, P. y GASTINGER, A. La alfabetización informacional como derecho humano. Anales de Documentación, 2012, vol. 15, nº 1. Disponible en: <http://revistas.um.es/analesdoc/article/view/147651>.
} 


\begin{abstract}
A clear line of argument can be set out to link the (passive) intellectual freedom rights offered by Article Nineteen of the United Nations' Universal Declaration on Human Rights, to a consequent responsibility on governments, professionals and civil society activists for the (active) creation of suitable conditions for the effective exercise of intellectual freedom. Commentators on media in society and socially responsible computing are also increasingly drawing conclusions of this kind and stressing the importance of Media Literacy and Computer Literacy. This line of argument naturally directs attention towards the rationale currently offered for Information Literacy as a focus of professional activity. Whilst there are many elaborately worked-out programmes for Information Literacy instruction, these have so far been largely derived from practical perceptions of need. However, broad statements such as the Prague Declaration 'Towards an Information Literate Society' of 2003 and the Alexandria Proclamation of 2005 can be seen as beginning to point towards a rationale for Information Literacy activities rooted in human rights, Article Nineteen in particular. The contention is that starting from a human rights perspective leads towards a strong, inclusive interpretation of Information Literacy. This subsumes Media Literacy, Computer Literacy, Web Literacy and, to a considerable extent, Civic Literacy into a model that serves human needs rather than the established priorities of information professionals. The value of this approach for both practice and research is stressed.
\end{abstract}

Keywords: Information literacy; human rights; intellectual freedom.

\title{
1. INTRODUCCIÓN
}

En este artículo intentamos analizar la posibilidad de la idea del acceso a la información como derecho humano para generar lo que nosotros pensamos que son argumentos convincentes a favor de la alfabetización informacional. Comenzaremos con alguna explicación del concepto del derecho a la información antes de pasar a desarrollar una línea de razonamiento que no es sólo académicamente interesante, sino que también puede ser de gran utilidad para aquellos profesionales que están implementando programas de alfabetización informacional.

El punto de partida es el Artículo 19 de la Declaración Universal de Derechos Humanos de las Naciones Unidas que establece que todo el mundo tiene derecho a la libertad de expresión. Lo importante es que este Artículo además deja claro que este derecho incluye el derecho al acceso a la información, que lo expresa como el derecho a "buscar, recibir y difundir información e ideas a través de cualquier medio y sin verse afectado por ningún tipo de limitación fronteriza” (Declaración Universal de los Derechos Humanos, 1948). Es difícil imaginar una declaración más significativa que justifique la labor del profesional de la información, aunque no se cite en los foros profesionales con la frecuencia que se merece. No obstante, la IFLA (Federación Internacional de Asociaciones de Bibliotecarios y Bibliotecas) anima a la profesión a encontrar inspiración en este Artículo, algo que lleva a cabo principalmente a través de la labor de su Comité para la Libertad de Acceso a la Información y Libertad de Expresión (FAIFE), de la cual el primer autor de este artículo fue presidente entre el 2003 y el 2009). 
Dicho comité ha llegado a la firme conclusión, aunque no totalmente específica, de que el Artículo 19 apunta a que en la política de las bibliotecas se debe enfatizar el acceso a la información. Aunque en un principio se podría haber considerado que FAIFE limitaba su trabajo a defender la libertad intelectual en aquellas bibliotecas donde este derecho se veía amenazado, en la actualidad este comité ha ampliado sus objetivos en gran medida.

Con objeto de promover el acceso a la información, FAIFE organizó, durante el período 2007-2009, talleres de trabajo en países en vías de desarrollo destinados a promover el uso activo de Internet y la difusión de información sobre el SIDA para el público en general. Además, durante el 2009 siguió desarrollando el concepto de igualdad social mediante el acceso a la información creando material sobre el acceso público a la información sanitaria, que utilizó por primera vez como proyecto piloto en los talleres de trabajo que organizó en agosto de ese mismo año en África oriental. También durante el 2009 desarrolló otro programa siguiendo los principios del "Manifiesto de la IFLA sobre transparencia, buena gobernanza y lucha contra la corrupción” (IFLA 2008) de FAIFE. El éxito de estos programas nos ha llevado a plantearnos que necesitamos buscar la justificación de nuestras actividades relacionadas con el fomento del acceso a la información en el Artículo 19. Además, este planteamiento nos puede llevar a otras implicaciones de tipo más general que se pueden deducir de este tipo de razonamiento, y la búsqueda de nuevas ideas para la alfabetización ha sido una consecuencia natural de ello.

El problema es que, aunque el Artículo 19 es una excelente fuente de inspiración (como cualquier declaración de tipo general sobre un derecho humano), éste no nos ofrece los argumentos necesarios que sustenten y desarrollen de modo concreto el derecho a la información. Argumentos que, por otro lado, nosotros debemos buscar y razonar por separado. Por ejemplo, una conclusión común es que las naciones necesitan leyes para la libertad de información o el acceso a ella que permitan al ciudadano a acceder por derecho a la documentación oficial del país. Más de 70 estados han aprobado leyes de este tipo que, aunque varían mucho en cuanto su eficacia, no obstante todas reflejan la idea de que el derecho a la información que se recoge en el Artículo 19 necesita este tipo de legislación para hacerlo realidad. Cuando decimos que el Artículo 19 tiene relevancia para los profesionales de la información, estamos, por lo tanto, siguiendo un proceso de razonamiento similar, aunque nosotros nos referimos a instituciones y servicios ya existentes (sistemas bibliotecarios y de información) y estamos ofreciendo una justificación retrospectiva de su trabajo. Esto es necesario ya que se puede afirmar que el trabajo de los profesionales de las bibliotecas y la información carece de argumentos rigurosos que lo justifiquen. La alfabetización informacional como nueva disciplina comparte este problema, como intentaremos demostrar más adelante. Una vez hayamos analizado los argumentos existentes que justifican la importancia de la alfabetización informacional, pasaremos a desarrollar una línea de argumentación a partir del Artículo 19 y a ofrecer algunas sugerencias de cómo ésta ofrece beneficios reales para la práctica de la formación en alfabetización informacional. 


\section{DEFINICIONES}

Los programas de formación que ofrecen las bibliotecas en relación a la alfabetización informacional se están multiplicando enormemente, aunque siguen basándose en las prioridades establecidas por los bibliotecarios. El lector puede comprobar la veracidad de esta afirmación consultando varios estudios internacionales (Andretta 2007; Curzon y Lampert 2007; Lau 2008) o haciendo una búsqueda en la web. Estos programas tienden a impartirse predominantemente en instituciones académicas, y consisten en clases teóricas y trabajo práctico con un enfoque muy didáctico. Es decir, se le da un gran énfasis a enseñar a los usuarios a utilizar las herramientas y servicios de la biblioteca, concentrándose en el uso del OPAC, cómo hacer búsquedas en las bases de datos y cómo localizar los libros en los estantes. Y aún más, podemos encontrar muchas similitudes entre los programas de formación de usuarios de los años 70 y 80 y los programas de alfabetización informacional de la actualidad. A veces esto es muy obvio, por ejemplo, “A la alfabetización informacional se le conoce con muchos nombres: orientación bibliotecaria, instrucción bibliográfica, formación de usuarios, habilidades para el uso de la información” (página web de la alfabetización informacional del Reino Unido).

Se podría afirmar que estas definiciones son, a lo sumo, posibles conceptos menores de lo que hoy día entendemos como alfabetización informacional. Cualquiera que haya tenido alguna relación con la formación de usuarios sabrá que la tendencia predominante consistía en hacer presentaciones monótonas de lo que ofrece la biblioteca, a lo que da acceso y cómo los usuarios pueden beneficiarse de todo ello. Con ello no sólo se aburría a los estudiantes que eran los destinatarios, sino que rara vez se tenían otras intenciones que las de crear "buenos" usuarios de la biblioteca. No se le prestaba mucha atención al pensamiento crítico y no se enseñaban realmente destrezas que ayudarán al usuario a seguir aprendiendo a lo largo de su vida de modo pudieran participar eficazmente en la sociedad. Incluso los bibliotecarios encargados de enseñarlos, a menudo parecían compartir la misma desilusión que tenían los estudiantes y solían mostrar poco entusiasmo o inspiración. Por todo esto, se puede decir que la formación de usuarios fue un proyecto fracasado que utilizaba una gran cantidad de recursos profesionales durante muchos años. Si la alfabetización informacional no es más que la formación de usuarios con un nuevo nombre, entonces los profesionales de la información no han progresado verdaderamente en la formación que le ofrecen a los usuarios.

Afortunadamente, el antiguo contenido de la formación de usuarios es sólo una parte de la mayoría de los programas de alfabetización informacional de hoy día, que prestan mayor atención al desarrollo de una actitud crítica hacia la información en todos sus formatos (Spencer y Millson-Martula 2009). Una forma rápida de ejemplificar esto sería realizar una campaña para convencer a los usuarios que una simple búsqueda en Google no es suficiente, si se quiere realizar un trabajo serio. Tomando como ejemplo un texto reciente (Bothma et al. 2006), podemos ilustrar esto. Esta publicación tiene una buena presentación con muchas capturas de pantallas de páginas de Internet que lo sitúan firmemente en el siglo XXI. Comienza con definiciones y una justificación de la 
alfabetización informacional que la acerca a la necesidad humana de información para tomar decisiones y realizar tareas y la relaciona con la idea de la formación permanente y las competencias de un ciudadano muy comprometido con su sociedad. Además, se presentan los modelos de los programas de alfabetización informacional desarrollados por Eisenberg et al. (2004) y Thomas (2004), se analiza el uso ético de la formación y los métodos de búsqueda y se evalúan las fuentes de información y otros temas relevantes de un modo ejemplar. ¿Es todo esto suficiente para describirlo como alfabetización informacional y distinguirlo de la formación de usuarios? Por supuesto que sí. En nuestra opinión, lo que a esto le falta es una línea de argumentación que lo relacione con un concepto más sólido que el de meramente enseñarle al individuo a desenvolverse en la sociedad de la información de una manera práctica.

Hay muchas definiciones de la alfabetización informacional, pero todas suelen tener una orientación práctica. Probablemente una de las definiciones más citadas es la de la Asociación Americana de Bibliotecarios (ALA 1989) que nos dice que "Para formarse en la alfabetización informacional, una persona debe ser capaz de reconocer cuándo necesita información y tener la capacidad de localizarla, evaluarla y usarla eficazmente”. Esta es una definición un poco anticuada y típicamente desde el punto de vista del bibliotecario, difiriendo muy poco de cómo se definía anteriormente la Formación de Usuarios, aunque seguirá citándose debido a la autoridad de la organización de donde proviene. Esta definición no refleja del todo bien que la Alfabetización Informacional consiste principalmente en un planteamiento individual y crítico hacia la información más que un conjunto de herramientas mecánicas que se deben aprender para obtener algún producto ideal (la información “correcta”). Podríamos continuar citando múltiples ejemplos de este tipo de definiciones pero no aportaríamos nada nuevo.

Hay una mejor definición que también es muy citada que considera la Alfabetización Informacional como "Un conjunto de competencias que un ciudadano informado de una sociedad de la información debe poseer para participar inteligentemente y activamente en la misma”. Esta definición es más rica y con una visión más amplia. No obstante, aunque se repite en muchas páginas web, a veces como una cita sin atribuir y otras como si fuese algo original, si se aporta una referencia, ésta parece llevarnos a una página web de la asociación EDUCAUSE, que desgraciadamente ya no existe. Por ello, dejamos la tarea de identificar la fuente original a aquellos lectores que posean una buena formación en Alfabetización Informacional o a cualquier otra persona que tenga la curiosidad de buscarla. Esta definición tiene en cuenta que la Alfabetización Informacional vas más allá de la habilidad de reconocer cuándo se necesita información, localizarla, evaluarla y utilizarla. La "Declaración sobre la Alfabetización Informacional para todos los Australianos” (ALIA 2001) expresa esto de forma más explícita y contundente, afirmando que la Alfabetización Informacional es una condición básica para la formación permanente, la creación de nuevos conocimientos, la adquisición de destrezas, la capacitación personal, vocacional, corporativa e institucional, la inclusión social, la participación ciudadana y la innovación y el espíritu emprendedor. 
Lo que vemos en definiciones de este tipo es que los autores se esfuerzan por encontrar una justificación de peso para una actividad educativa que el sentido común nos dice que obviamente vale la pena. Necesitan esta justificación por varias razones, algunas de las cuales debatiremos en otro apartado. La principal necesidad es convencer a la gente a la que instruyen de que la Alfabetización Informacional es importante para ellos, aunque el argumento del sentido común puede ser suficiente ya que es poco probable que la gente que recibe formación en Alfabetización Informacional necesite justificaciones basadas en la teoría. No obstante, nosotros sostenemos que incluso en estas circunstancias merece la pena fortalecer aún más este argumento basándolo en el derecho a la información. No estamos completamente solos en este argumento ya que los inicios de este planteamiento basado en el derecho a la información se pueden encontrar en las declaraciones sobre la Alfabetización Informacional realizadas en los primeros años del nuevo milenio y sobre ellos hablaremos en el siguiente apartado.

\section{APARICIÓN DEL ARGUMENTO DE LOS DERECHOS HUMANOS}

Podemos ver indicios de una actitud más fundamental sobre la alfabetización informacional cuando, por ejemplo, el Proyecto de Alfabetización Informacional Escocés (2004-2009) hacía referencia a la "alfabetización informacional como un derecho civil”. También se puede observar claramente esta tendencia en algunas declaraciones internacionales, que han comenzado a afianzar la idea de que la alfabetización informacional no es simplemente una necesitad, sino un derecho humano básico que promueve la inclusión social en todos los países. Por ejemplo, la Declaración de Praga "Hacia una Sociedad de la Alfabetización Informacional” propuso los principios básicos que dicen, entre otras cosas, que la alfabetización informacional "es un prerrequisito para participar eficazmente en la Sociedad de la Información y forma parte del derecho humano básico de la formación permanente” (Declaración de Praga, 2003). Lo importante de esto es que se menciona el derecho a la formación permanente, al que se le añade la alfabetización informacional. Esta declaración continúa diciendo que la alfabetización informacional "debería formar parte integral de la Educación para Todos, que puede contribuir de modo fundamental al logro de los Objetivos de Desarrollo del Milenio de las Naciones Unidas y al respeto de la Declaración Universal de los Derechos Humanos”.

Aunque la idea de que la alfabetización informacional puede contribuir hacia el logro de los Objetivos de Desarrollo del Milenio es una extensión natural de la línea de razonamiento manifestada en la Declaración Australiana mencionada anteriormente, la referencia a los Derechos Humanos es algo nuevo. La redacción es ambigua pero parece sugerir que las personas formadas en la alfabetización informacional respetarán los derechos de los demás, más que para ellos la alfabetización es un derecho, aunque, no obstante, la interrelación es significativa. La Cumbre Mundial sobre la Sociedad de la Información (WSIS) estableció una interrelación similar en varias de sus declaraciones como, por ejemplo, en la Declaración de Principios y en la Declaración de la Sociedad Civil, en las que resalta la importancia del Artículo 19 de la Declaración Universal de los 
Derechos Humanos. En concreto, la Declaración de la Sociedad Civil nos dice que el Artículo 19 "constituye una condición esencial para las sociedades de la comunicación e información basadas en los derechos humanos (WSIS 2003). Una vez más, la redacción del documento no deja especialmente claro la importancia de esa interrelación, aunque la idea de que existe una conexión entre los dos está presente.

Para tener una definición más clara de esta idea debemos recurrir a la Proclamación de Alejandría. No obstante y puesto que su objetivo era influir en los debates y las conclusiones de la Cumbre Mundial, podemos pensar que ésta última no llegó a captar bien algunas de las ideas expresadas en la Proclamación, ya que ésta describe la Alfabetización Informacional y la Formación Permanente como las "guías de la Sociedad de la Información que iluminan el camino hacia el desarrollo, la prosperidad y la libertad”. También declara que la Alfabetización Informacional, como la base sobre la que descansa la Formación Permanente, "faculta a las personas para conseguir sus objetivos personales, sociales, laborales y educativos" y es "un derecho humano básico en un mundo digital” (Proclamación de Alejandría 2005). El hecho de que ni la declaración australiana mencionada anteriormente que, según afirma, se inspira en la Proclamación, ni la Cumbre Mundial recogen esta línea de argumentación nos sugiere que era demasiado avanzada para su tiempo. Hasta la Proclamación de Alejandría, las declaraciones mencionadas anteriormente no establecen una relación clara entre el derecho a la información y la Alfabetización Informacional, salvo de pasada y sin entrar en más detalle. En el siguiente apartado nos dedicaremos a argumentar más claramente la relación existente.

\section{EL ARGUMENTO DE LOS DERECHOS HUMANOS}

Pensamos que se puede desarrollar una línea clara de argumentación que vincule los derechos de la libertad intelectual (argumento pasivo) contenida en el Artículo 19 con una responsabilidad consecuente por parte de los gobiernos, los profesionales y los activistas de la sociedad civil para la creación (argumento activo) de las condiciones adecuadas para el ejercicio eficaz de la libertad intelectual. Lo que se necesita para darle más peso es pruebas de que algún gobierno o gobiernos en alguna parte del mundo también han considerado que éste es un argumento válido y, ciertamente, hay un ejemplo de ello. Podemos encontrar una interpretación especialmente sólida de las implicaciones del Artículo 19 en el nuevo Artículo 100 de la Constitución Noruega promulgado en el 2005. Este no sólo hace referencia al derecho de acceso a los documentos del gobierno, sino que exige a las autoridades estatales que creen las condiciones que faciliten el diálogo abierto entre una población informada. La naturaleza de estas "condiciones” no se especifican claramente, aunque Noruega las ve como un conjunto de disposiciones que afectan no sólo a las escuelas, sino también a las "instituciones culturales públicas como las universidades, bibliotecas, museos, la radio y la televisión y organismos similares así como a una gran diversidad de instituciones privadas reguladas y apoyadas por las autoridades públicas como los medios de comunicación, las editoriales, teatros, cines y otros lugares públicos de encuentro” (Noruega 2005). Estas instituciones, protegidas y 
administradas eficazmente, deberían proporcionar las condiciones propicias para el desarrollo de la libertad de expresión en el ámbito público.

El concepto de “ámbito público” nos lleva a una fuente especialmente fructífera de ideas para los profesionales de la información como son los trabajos de Jürgen Habermas. La teoría del ámbito público (Öffentlichkeit), en el que los individuos puedan intercambiar conocimiento e ideas se ha identificado generalmente con su nombre (Habermas 1989). Su argumento comienza con la identificación de una cultura "representativa" dominante hasta el siglo XVIII en la que los gobernantes imponían su voluntad y su imagen sobre la conciencia de sus súbditos. Por el contrario, ve la aparición en este mismo siglo de foros que estaban fuera del control del estado, principalmente en Inglaterra y en los Estados Unidos de América, que acababan de independizarse, en los que las personas tenían la posibilidad de desarrollar sus propias ideas y expresarlas en debates abiertos, valiéndose de los medios impresos como periódicos, panfletos y libros para divulgarlas. Muestra de ello son, ciertamente, los miles de clubs y sociedades que existieron entonces y que iban desde la eminente Royal Society fundada en el 1662 hasta las humildes asociaciones de jardineros y hortelanos en las provincias (Clark 2001). En un clima social e intelectual de este tipo, la libertad de expresión era la norma y el cambio político que experimentó Gran Bretaña en el siglo XIX es consecuencia directa de ello. Quizás la característica más distintiva del dominio público del siglo XVIII en Gran Bretaña es que floreció en un clima de abandono en el que el estado no se preocupaba de suprimir un fenómeno que amenazaba las estructuras y las creencias existentes.

La tesis de Habermas es que la esfera pública ha decaído con posterioridad bajo la presión de una diversidad de fuerzas, en la que la influencia de los medios de comunicación y de la cultura del consumo de información es crucial. La toma de decisiones, que había estado cerca de los participantes en el dominio público, se ha traslado a los burócratas y a unos gobiernos con demasiada libertad de actuación que están muy alejados del ciudadano medio, por lo que es necesario renovar el dominio público para que el desarrollo de la opinión pública pueda florecer de nuevo. El nuevo artículo 100 promulgado por el gobierno noruego se puede ver como una especie de respuesta a esta propuesta de Habermas, aunque no especifica en detalle cómo la infraestructura de la esfera pública puede verdaderamente ayudar a desarrollar la vida intelectual activa que necesita para existir, por muy bien que el gobierno de un estado progresista pueda fomentarlo. Las instituciones como las bibliotecas, por citar sólo el ejemplo más relevante, no pueden por su mera existencia garantizar un población que participe activamente con ideas y capaz de examinar críticamente los mensajes que les llegan de todos los medios de comunicación.

Nuestra conclusión es que existe una necesidad demostrable de garantizar que la población posea las destrezas necesarias para hacer realidad los derechos que nos ofrece el Artículo 19. Cada vez más, los analistas del papel de los medios de comunicación y de uso de la informática para beneficio de la sociedad están llegando a conclusiones de este tipo, destacando la importancia de la enseñanza de la Alfabetización Mediática y en 
Informática. Los profesionales de la información también han llegado a una postura muy similar, incluidos aquellos que han organizado, presentado y evaluado los Talleres de FAIFE, cuyas actividades apuntan claramente a los programas de Alfabetización Informacional como un requisito necesario. Estos programas intentan solventar el problema de que las bibliotecas puedan estar ofreciendo acceso a la información a un público que, de hecho, no está plenamente capacitado para hacer un buen uso de él. Sin un buen nivel de Alfabetización Informacional, la abundante cantidad de información a la que podemos acceder hoy día puede simplemente confundir y engañar. Al referirnos al Artículo 19 y analizar de cerca sus implicaciones, como hemos hecho aquí, los argumentos a favor de la Alfabetización Informacional quedan claros.

\section{POSIBILIDADES DEL ENFOQUE DE LOS DERECHOS HUMANOS}

Si podemos afirmar que la necesidad de la formación del individuo en Alfabetización Informacional es el resultado lógico del derecho humano a la información, entonces los programas de desarrollo de la alfabetización son la implementación de ese derecho. No obstante, como ya hemos sugerido, esta conclusión significa que el tipo de Alfabetización Informacional al que hemos llegado según nuestro planteamiento no es propiedad exclusiva de una profesión, como la del bibliotecario. Por ello, necesitamos reconocer que, al menos, existen distintos tipos definiciones y grandes grupos de programas al servicio de la Alfabetización Mediática, Alfabetización en Informática, Alfabetización Digital, Alfabetización Web, Alfabetización Crítica y Alfabetización Cívica (Lankshear y Knobel 2008). Otros pueden muy bien haber identificado y desarrollado otros tipos de alfabetizaciones, pero esta lista es lo suficientemente amplia como para ilustrar tanto los puntos que tienen en común como sus diferencias. Si comenzamos, como han hecho los bibliotecarios, con la Formación de Usuarios y la transformamos posteriormente a Alfabetización Informacional, la relación con cualquier otra versión de Alfabetización es más o menos un añadido que no pasa de ser interesante.

Si, por el contrario, comenzamos con el concepto de acceso a la información como derecho humano, esto nos lleva al área general de las alfabetizaciones y los programas que las apoyan y, lo que es más, no necesitaremos tratarlas como si las distinciones existentes entre ellas las hicieran realmente áreas distintas. La conclusión a la que llegamos al tratar del área de la Alfabetización Informacional desde este punto de vista es que el individuo necesita un amplio conjunto de destrezas escogidas por él mismo referentes a los diversos formatos y soportes para hacer realidad su derecho a la información. Esto está en relación con la forma de buscar y utilizar la información por las personas, que es global y no se ve necesariamente limitada por un tipo determinado de formato o institución y que, en potencia, les ofrece los tipos de ayuda que necesitan, más que lo que un grupo específico de profesionales piensan que deberían necesitar.

La esencia de este argumento es que si partimos de la perspectiva de los derechos humanos, esto nos conduce a una interpretación sólida e inclusiva de la Alfabetización Informacional, de modo que ésta engloba a todas las formas específicas de alfabetización 
promovidas por los diferentes grupos profesionales, aunque compartiendo el mismo principio. Esta interpretación puede ser discutible, pero el concepto tiene un nivel de generalidad que le permite englobar todos los tipos de alfabetización que se hayan identificado, descrito y enseñado. Para aclarar este punto, necesitamos presentar un breve resumen de algunas de las características que nosotros creemos conforman esta gran categoría. La Alfabetización Informacional, tal y como se imparte en las bibliotecas, abarca la diversa variedad de fuentes (desde el formato impreso al digital) que ofrece una biblioteca moderna y de calidad, aunque en los otros tipos de alfabetización el énfasis varía yendo de los más específico a lo (probablemente) más general.

En primer lugar, mencionaremos la Alfabetización Mediática que es la interpretación de lo que recibimos de los periódicos y otras publicaciones, la radio, el cine, la televisión y las noticias en Internet. La Oficina de Comunicaciones del Reino Unido (Ofcom 2009) la define como "la habilidad para acceder, entender y crear comunicaciones en una diversidad de contextos”. De todos los tipos de alfabetización, la Alfabetización Mediática es posiblemente la que más está de moda. Entender las funciones de los propietarios de los medios, los editores, los periódicos y ser consciente de la forma confusa en la que lo que dicen parece "correcto" forma parte de esto, aunque también lo es la interpretación de los mensajes publicitarios y promocionales, que es algo omnipresente en el mundo de los medios de comunicación. Se ha escrito muchísimo, de forma especulativa y propagandista, sobre la influencia de los medios de comunicación en el comportamiento como, por ejemplo, los estudios sobre la pornografía y sus efectos en el comportamiento sexual. La Alfabetización Mediática es más una disciplina académica que una enseñanza práctica, aunque los programas de estudios universitarios enseñan a los estudiantes a entender la forma en la que se puede manipular el lenguaje y las imágenes para influir en las ideas, la opinión y (posiblemente) el comportamiento.

La Alfabetización en Informática, la Alfabetización Digital y la Alfabetización Web son tres componentes que se solapan pero razonablemente distintos, relacionados con la parte electrónica del mundo de la información. La Alfabetización en Informática se ocupa en gran parte de la formación en el uso de los programas informáticos (principalmente los más básicos como los procesadores de textos, las bases de datos y las hojas de cálculo), las aplicaciones y dispositivos periféricos como impresores y escáneres. Esto implica la capacidad de saber usar y manejar los ordenadores como máquinas para el procesamiento de la información (Horton 2008). Las clases para ayudar a los niños, adultos y personas mayores a obtener el conocimiento básico necesario para poder utilizar los medios informáticos a su alcance representan probablemente las más comunes en la enseñanza de la alfabetización. Acertadamente, en estas clases rara vez se enseña el funcionamiento interno de los ordenadores y sus programas. La Alfabetización Digital generalmente va un paso más allá de la Alfabetización en Informática y se ocupa mucho más de localizar, organizar, entender, evaluar y crear contenidos con la ayuda de la informática. El gobierno de Nueva Zelanda define la Alfabetización Digital como "la habilidad de utilizar la tecnología digital, las herramientas de comunicación o las redes para localizar, usar y crear información” (Definición de Alfabetización Digital de Nueva Zelanda, sin fecha). 
Las definiciones de Alfabetización Digital ponen cierto énfasis en la comprensión del funcionamiento del hardware y también prestan atención a los dispositivos digitales como teléfonos móviles e Internet. La Alfabetización Web, como su nombre indica, se ocupa de la evaluación e interpretación del contenido de Internet y del uso de aplicaciones como los blogs, los wikis y las redes sociales.

Menos conocido puede ser el concepto de Alfabetización Cívica, que es absolutamente fundamental para los ciudadanos de una sociedad democrática y para aquellos que esperan iniciar el cambio e introducir una democracia más sólida (Milner 2002). Se basa en una alfabetización práctica e incluye la interpretación tanto de los medios de comunicación como de los mensajes de propaganda ideológica y política. Como ejemplo de la forma en la que se utiliza este término, podemos mencionar la sugerencia que se ha hecho de que los problemas de los campamentos del millón de Personas Desplazadas Internamente (PDI) del norte de Uganda se pueden abordar mediante programas de Alfabetización Cívica. Estos problemas se deben a que "el analfabetismo y la pobreza son enormes barreras para la comunicación. La incapacidad de leer y escribir ocasiona graves inconvenientes al intentar comunicarse con las autoridades u obtener información del gobierno local. Los folletos y carteles sólo son buenos si la persona a la que van dirigidos sabe leer” (Buttedahl y Nkurunziza 2005, 11) y nosotros añadiríamos “y si puede averiguar quién está tratando decirte qué y por qué”.

Por últimos, tenemos la Alfabetización Crítica, un término aun más amplio que la Alfabetización Informacional en sí, cuyas raíces se encuentran en las ideas educativas de Shor (1980) que identifican claramente al espíritu crítico como el elemento central de todo el proceso educativo o, según la terminología moderna, el proceso de aprendizaje. En sus propias enseñanzas, Shor trató de enseñar a los estudiantes a ir más allá del significado 'superficial' de la información e ideas a las que estaban expuestos con objeto de entender mejor las causas, contexto e ideología de todos los tipos de comunicación. Este tipo de planteamiento crítico es la base de todos los tipos de alfabetizaciones descritos anteriormente, lo que parece sugerirnos que deberíamos sustituir la Alfabetización Informacional por la Alfabetización Crítica como el término general que lo engloba a todo. No obstante, existen dos problemas con este planteamiento. La primera es la asociación que se establece entre Alfabetización Crítica y la enseñanza como opuesta al aprendizaje y, en segundo lugar, es la connotación de crítica literaria que el término “crítico” conlleva. Debido a esto, quizás un término ideal podría ser la Alfabetización Informacional Crítica, pero nuestro argumento principal es que todas estos tipos de alfabetizaciones se derivan naturalmente del Artículo 19, según lo hemos descrito anteriormente. Todas ellas deben considerarse, en primer lugar, como elementos de una categoría más amplia (que, según nosotros, es la Alfabetización Informacional) y, a continuación, importantes por sí mismas como enriquecimientos especializados de esa categoría. 


\section{CONCLUSIÓN}

En conclusión, es necesario ofrecer una respuesta a la pregunta ¿Es esta una línea de argumentación útil o se trata simplemente de una manera de teorizar sobre la Alfabetización Informacional (más o menos, teorizar por teorizar)? La respuesta sería que un planteamiento global y unificador como el que hemos propuesto es más eficaz para el individuo que la creación de diferentes programas para los diversos tipos de alfabetizaciones, tal y como ya proporcionan los diferentes grupos profesionales en distintos contextos institucionales. Este planteamiento ofrece oportunidades para la cooperación entre los diferentes tipos de alfabetizaciones con objeto de promover la Alfabetización Informacional y mejorar el diseño de los programas educativos. Con esto lo que queremos decir es que, por ejemplo, este planteamiento debería animar a los bibliotecarios a ir más allá de los aspectos relacionados con el uso de la biblioteca, que es lo que normalmente hacen. No obstante, lo más importante de este planteamiento es que podemos desarrollar argumentos coherentes basados en los principios básicos como los derechos humanos para intentar convencer a los responsables de la financiación y administración del valor de estos programas, que van mucho más allá de las cuestiones meramente técnicas.

También diríamos que un concepto global de Alfabetización Informacional está relacionado con otros requisitos que podemos identificar analizando la importancia de un derecho a la información. Por dar sólo un ejemplo de esto, ya hemos identificado la manera en la que un derecho a la información necesita apoyarse en el acceso, tal y como lo posibilitan las leyes de Libertad de Información. Estas leyes no son fáciles de usar por una persona normal para obtener buenos resultados, por lo que necesita de la ayuda de personas formadas en la alfabetización, como son los profesionales de la información, que puedan enseñarle a formular preguntas según la legislación existente sobre la libertad de información. Esto nos lleva de nuevo al trabajo de FAIFE en educación y defensa de los intereses de los ciudadanos que se inspira en el Artículo 19 y se materializa en talleres de trabajo que también tienen implicaciones para las actividades profesionales de los formadores de la Alfabetización Informacional. Sus talleres sobre el Acceso a Internet, el Acceso Público a la Información Sanitaria y la Transparencia se mencionaron al principio de este artículo. Todos estos programas alertan a los bibliotecarios y otros profesionales de la información sobre áreas determinadas en las que pueden ayudar al usuario mediante la información. No obstante, no se trata simplemente de informar al ciudadano, sino que lo que los profesionales deberían hacer es ayudar a la gente a desarrollar su capacidad de comprender e interpretar la información.

Para finalizar, debemos hablar de la idea que hemos presentado en este artículo desde la perspectiva de la investigación y el desarrollo. Hemos dicho que si partimos de la idea del derecho a la información entonces necesitaremos programas de Alfabetización Informacional, en el sentido más amplio posible. No obstante, este planteamiento no responde a todas las cuestiones relacionadas con la forma, ubicación, contenido, estructura y método de esos programas, ya que nosotros hemos ofrecido simplemente una 
racionalización a posteriori de lo que ya se está haciendo. Por consiguiente, los investigadores necesitan responder preguntas como qué posibilidades existen de diseñar programas que enseñen al individuo las destrezas genéricas que son comunes a los diferentes tipos de alfabetizaciones. También deben estudiar el entorno en el que la gente vive, trabaja, estudia y disfruta de su tiempo libre con el fin de poder identificar nuevas posibilidades para las actividades relacionadas con la Alfabetización Informacional. A partir de este tipo de investigación aplicada, podremos desarrollar programas de Alfabetización Informacional que puedan ayudar al desarrollo personal del individuo a través del acceso y uso de la información. Partiendo de una línea de razonamiento basada en la idea del derecho a la información, los profesionales disponemos de enormes posibilidades por explotar en el campo de la Alfabetización Informacional.

\section{BIBLIOGRAFÍA}

ALA. 1989. Presidential Committee on Information Literacy. Final Report. Chicago: American Library Association.

Alexandria Proclamation. 2005. URL: http://archive.ifla.org/III/ wsis/BeaconInfSoc.html [accessed 4 July 2010].

ALIA. 2001. Statement on Information Literacy for all Australians. URL: http://www.alia.org.au/policies/information. literacy.html [accessed 15 September 2009].

Andretta, S. 2007. Change and challenge: Information literacy for the 21st century. Adelaide: Auslib Press.

Bothma, T., E. Cosijn, I. Fourie, and C. Penzhorn. 2006. Navigating Information Literacy: Your Information Society Survival Toolkit. Cape Town, South Africa: Pearson Education South Africa.

Buttedahl, P., y D. Nkurunziza. 2005. An assessment of civic literacy in Uganda's local government. Kampala: Peace and Conflict studies, Makerere University.

Clark, P. 2001. British clubs and societies, 1580-1800. Oxford: University Press.

Curzon, SC., y LD. Lampert. 2007. Proven strategies for building an information literacy program. New York: Neal-Schuman Publishers Inc.

Eisenberg, MB., CA. Lowe, and KL. Spitzer. 2004. Information literacy: essential skills for the information age. 2d ed. Westport, CT: Libraries Unlimited.

Habermas, J. 1989. The Structural Transformation of the Public Sphere. Trans. T. Burger. Cambridge: Polity Press.

Horton Jr, FW. 2008. Understanding Information Literacy: A Primer. UNESCO. URL: http://unesdoc.unesco.org/images/ 0015/001570/157020e.pdf [accessed 21 January 2009].

IFLA. 2008. IFLA Manifesto on Transparency, Good Governance and Freedom from Corruption. URL: http://www.ifla. org/en/publications/ifla-manifesto-on-transparencygood-governance-and-freedom-from-corruption [accessed 15 June 2009].

Lau, J. 2008. Information Literacy: International Perspectives. IFLA Publications 131. München: K.G. Saur Verlag. 
Lankshear, C., y M. Knobel. 2008. Digital literacies: concepts, policies and practices. New York: Peter Lang Publishing Inc.

Milner, H. 2002. Civic literacy: how informed citizens make democracy work. Hanover: University Press of New England.

New Zealand Digital Literacy Definition. URL - http://www. digitalstrategy.govt.nz/Resources/Glossary-of-Key-Terms/ [accessed 27 September 2009].

Norway. 2005. There shall be freedom of expression: proposed new Article 100 of the Norwegian Constitution. Official Norwegian Reports NOU 1999: 27, 39. Oslo: Ministry of Justice and the Police.

Ofcom. 2009. Report of the Digital Britain Media Literacy Working Group. URL: http://www.ofcom.org.uk/advice/ media_literacy/media_lit_digital_britain/digitalbritain.pdf [accessed 27 September 2009].

Prague Declaration. 2003. Towards an information literate society. URL http://portal.unesco.org/ci/en/files/19636/

11228863531PragueDeclaration.pdf/PragueDeclaration.pdf [accessed 21 January 2009].

Scottish Information Literacy Project. 2004-2009. URL: http:// www.caledonian.ac.uk/ils [accessed 17 September 2009].

Shor, I. 1980. Critical Teaching and Everyday Life. Chicago: University of Chicago Press.

Spencer, J., y C. Millson-Martula. 2009. Critical thinking within the library program. London: Routledge.

The Universal Declaration of Human Rights. 1948. URL: http:// www.un.org/en/documents/udhr/ [accessed 17 June 2009].

Thomas, N. 2004. Information literacy and information skills instruction: applying research to practice in the school library media center. Westport, CT: Libraries Unlimited.

UK Information Literacy Website. URL: http://www. informationliteracy.org.uk/information_literacy.aspx [accessed June 16, 2009].

WSIS. 2003. Shaping Information Societies for Human Needs. Civil Society Declaration to the World Summit on the Information Society. URL: http://www.itu.int/wsis/docs/geneva/ civil-society-declaration.pdf [accessed 21 January 2009]. 